\title{
BOUNDING THE LOCATING-TOTAL DOMINATION NUMBER OF A TREE IN TERMS OF ITS ANNIHILATION NUMBER
}

\author{
WenJie Ning \\ College of Science \\ China University of Petroleum (East China) \\ Qingdao 266580, China \\ e-mail: ningwenjie-0501@163.com \\ MEI LU \\ Department of Mathematical Sciences \\ Tsinghua University, Beijing 100084, China \\ e-mail: mlu@math.tsinghua.edu.cn \\ AND \\ KUN WANG \\ School of Mathematical Sciences \\ Anhui University, Hefei 230601, China \\ e-mail: wangkun26@163.com
}

\begin{abstract}
Suppose $G=(V, E)$ is a graph with no isolated vertex. A subset $S$ of $V$ is called a locating-total dominating set of $G$ if every vertex in $V$ is adjacent to a vertex in $S$, and for every pair of distinct vertices $u$ and $v$ in $V-S$, we have $N(u) \cap S \neq N(v) \cap S$. The locating-total domination number of $G$, denoted by $\gamma_{t}^{L}(G)$, is the minimum cardinality of a locating-total dominating set of $G$. The annihilation number of $G$, denoted by $a(G)$, is the largest integer $k$ such that the sum of the first $k$ terms of the nondecreasing degree sequence of $G$ is at most the number of edges in $G$. In this paper, we show that for any tree of order $n \geq 2, \gamma_{t}^{L}(T) \leq a(T)+1$ and we characterize the trees achieving this bound.
\end{abstract}

Keywords: total domination, locating-total domination, annihilation number, tree.

2010 Mathematics Subject Classification: 05C69. 


\section{REFERENCES}

[1] M. Blidia and W. Dali, A characterization of locating-total domination edge critical graphs, Discuss. Math. Graph Theory 31 (2011) 197-202. doi:10.7151/dmgt.1538

[2] M. Chellali, On locating and differentiating-total domination in trees, Discuss. Math. Graph Theory 28 (2008) 383-392. doi:10.7151/dmgt.1414

[3] M. Chellali and N. Jafari Rad, Locating-total domination critical graphs, Australas. J. Combin. 45 (2009) 227-234.

[4] X. Chen and M.Y. Sohn, Bounds on the locating-total domination number of a tree, Discrete Appl. Math. 159 (2011) 769-773. doi:10.1016/j.dam.2010.12.025

[5] W.J. Desormeaux, T.W. Haynes and M.A. Henning, Relating the annihilation number and the total domination number of a tree, Discrete Appl. Math. 161 (2013) 349-354. doi:10.1016/j.dam.2012.09.006

[6] W.J. Desormeaux, M.A. Henning, D.F. Rall and A. Yeos, Relating the annihilation number and the 2-domination number of a tree, Discrete Math. 319 (2014) 15-23. doi:10.1016/j.disc.2013.11.020

[7] O. Favaron, M.A. Henning, J. Puecha and D. Rautenbach, On domination and annihilation in graphs with claw-free blocks, Discrete Math. 231 (2001) 143-151. doi:10.1016/S0012-365X(00)00313-7

[8] J.R. Griggs and D.J. Kleitman, Independence and the Havel-Hakimi residue, Discrete Math. 127 (1994) 209-212. doi:10.1016/0012-365X(92)00479-B

[9] T.W. Haynes, M.A. Henning and J. Howard, Locating and total dominating sets in trees, Discrete Appl. Math. 154 (2006) 1293-1300. doi:10.1016/j.dam.2006.01.002

[10] M.A. Henning and N. Jafari Rad, Locating-total domination in graphs, Discrete Appl. Math. 160 (2012) 1986-1993. doi:10.1016/j.dam.2012.04.004

[11] L. Jennings, New Sufficient Condition for Hamiltonian Paths (Ph.D. Dissertation, Rice University, 2008).

[12] C.E. Larson and R. Pepper, Graphs with equal independence and annihilation numbers, Electron. J. Combin. 18 (2011) \#P180.

[13] R. Pepper, Binding Independence (Ph.D. Dissertation, University of Houston, 2004).

[14] R. Pepper, On the annihilation number of a graph, in: Recent Advances in Applied Mathematics and Computational and Information Sciences, Vol. I, K. Jegdic, P. Simeonov, V. Zafiris (Ed(s)), (WSEAS Press, 2009) 217-220.

Received 28 November 2016 Accepted 6 May 2017 